\title{
Comparative Analysis of Infrastructure and Ad-Hoc Wireless Networks
}

\author{
Shanna Li $i^{1, a}$ \\ ${ }^{1}$ Information Technology Center, Beijing Jiaotong University, 100044 Beijing, China
}

\begin{abstract}
The paper introduces the difference between infrastructure and ad hoc mode in the $802.11 \mathrm{Wi}-\mathrm{Fi}$ framework. We evaluate and compare the performance of ad hoc versus infrastructure through a series of experiments. We evaluate the performance depending of the mobility of the nodes and range of the area covered by the nodes.
\end{abstract}

\section{Introduction}

The IEEE 802.11 has two basic modes of operation: ad hoc mode and infrastructure mode (STA-AP). In ad hoc mode, each node transmits data directly to other nodes without access point. In infrastructure mode, all communication needs to go through the access point (AP) which connects wireless devices (Stations) to wired network. Under different scenarios, which is better, ad hoc mode or STA-AP mode ? In this case, 'better' means that more packets server node received in the same period of time when the same application was installed on the client node. In this paper, we can draw conclusions from a series of experiments using NS-3 simulator. We'll compare infrastructure and ad hoc networks to see which network can work best. The main method is comparing the number of the received packets through FlowMonitor using FlowMonitorHelper.

\section{Ad hoc and Infrastructure Wireless Networks}

\subsection{Ad hoc Wireless Networks}

There is no access point in ad hoc network. Nodes are within range of each other they can communicate directly with no access point. [1] Moreover, Nodes organize themselves into a network and route among themselves. Each node participates in routing by forwarding data for other nodes.[2]

In the Windows operating system, ad-hoc is a communication mode (setting) that allows computers to directly communicate with each other without a router. [3]

\subsection{Infrastructure Wireless Networks}

In infrastructure mode (STA-AP), all communication needs to go through the access point (AP), and access point plays key role in connecting wireless devices (Stations) to wired network.

Let's take two laptops positioned next to each other as an example. They can be connected to the same wireless network, but they are not directly communicating with each other. What happens is, one device sends packets to the access point and the packets are sent to the other laptop. To connect all the devices, you'd need an infrastructure mode network with a central access point. [4]

\section{NS-3 Introduction}

\subsection{NS-3}

Network Simulator 3(NS-3) is free software, licensed under the GNU GPLv2 license, and is publicly available for research, development, and use.[5]NS-3 is modular design network simulator can be connected to a real network. It has logging facility for debugging and tracing for getting output. Most of the users focus on wireless simulation includes models for $\mathrm{Wi}-\mathrm{Fi}$ and routing protocols such as AODV and OLSR. NS-3 is split over number of modules having one or more models for real network device and protocols.[6]

The first is to download and build an official release from the main web site. After we finished downloading and building ns-3, we wrote some scripts that will create a WiFi link between the nodes and generate traffic between the nodes.

\subsection{FlowMonitor}

FlowMonitor is a network monitoring framework for the NS-3 which can be easily used to collect and store network performance data from a NS-3 simulation instead of WireShark. FlowMonitor can automatically detect all flows passing through network. NS-3 provides

\footnotetext{
a Corresponding author: 1sn@bjtu.edu.cn
} 
FlowMonitorHelper class to create FlowMonitor easily. The helper will take care of about everything.

\section{Simulation experiments}

\subsection{Experimental purpose}

A series of experiments have been done using NS-3 under different scenarios. Meanwhile, we made comparative analysis between ad hoc mode and infrastructure mode.

We mainly focused on the packets server node received in the same period of time when the same application was installed on the client node.

\subsection{3 nodes topology}

There are 3 nodes topology which is equilateral triangle.

Each node will be installed ad hoc mode and infrastructure mode. Application is OnOff using OnOffHelper. Generate traffic from n0 to n2, as follows.

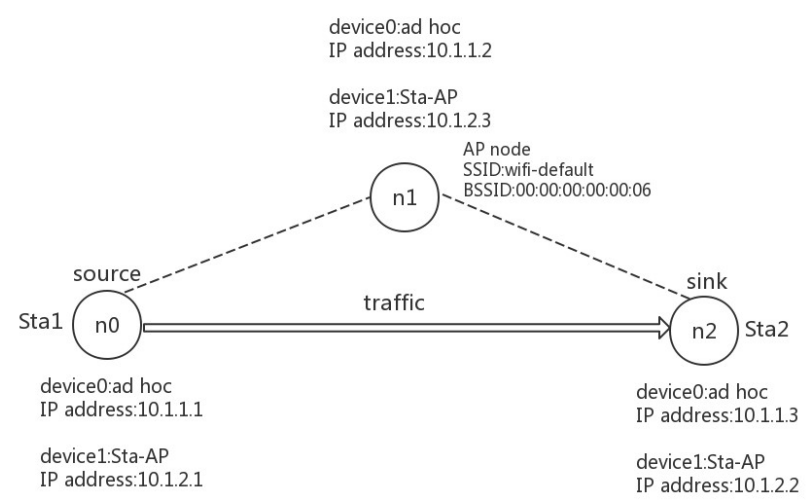

Figure 1. 3 nodes topology.

We can see from the figure 2 that ad hoc mode is better within a certain distance. STA-AP mode is better beyond a certain distance.

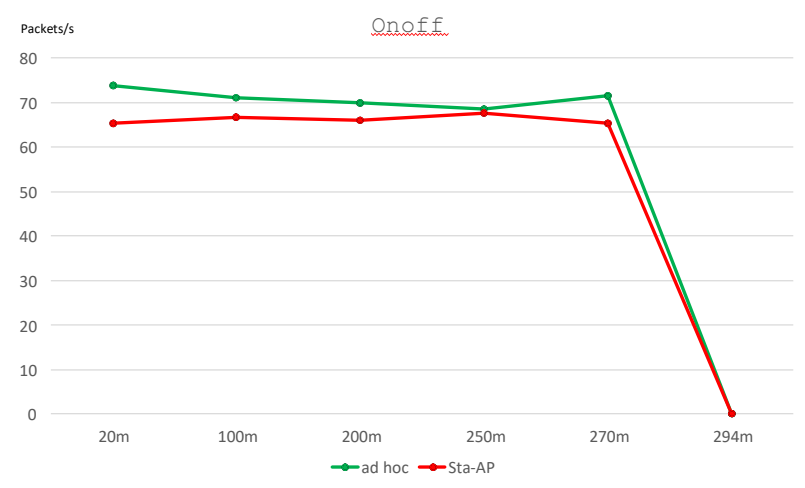

Figure 2. packets/s with different distance.

Since STA-AP mode is better beyond a certain distance, if we try to add routing on the nodes in ad hoc mode, we can find ad hoc mode is better than STA-AP mode after adding AODV routing protocol.STA-AP mode is better than ad hoc mode after adding OLSR routing protocol.

\subsection{5 nodes topology}

We will simulate topology like figure 3 in different simulator.Each node will be installed ad hoc protocol or STA-AP Protocol.The nodes will be installed routing protocol when they are in ad hoc mode.Generate traffic flow1 from $\mathrm{n} 0$ to $\mathrm{n} 2$. Generate traffic flow 2 from $\mathrm{n} 3$ to $\mathrm{n} 4$

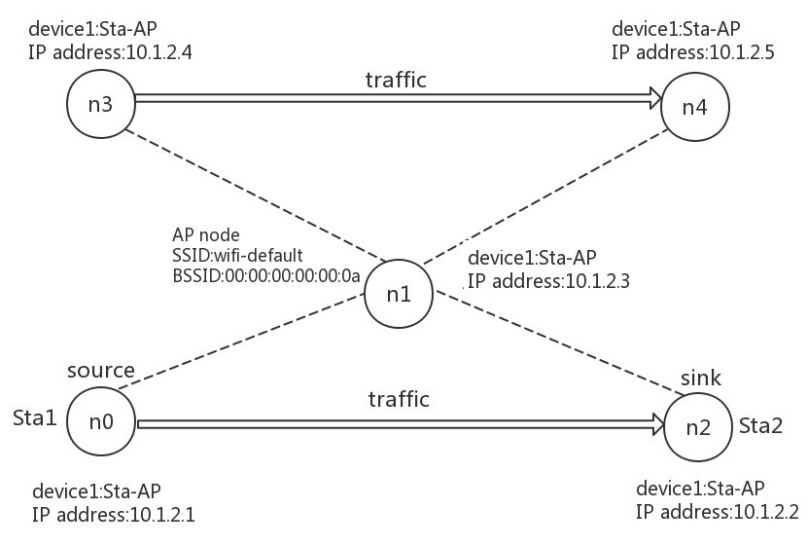

Figure 3. 5 nodes topology.

We can draw conclusions from experiment that no matter AP node is static or moving, the number of packets received in ad hoc mode is always two times that of STAAP mode.

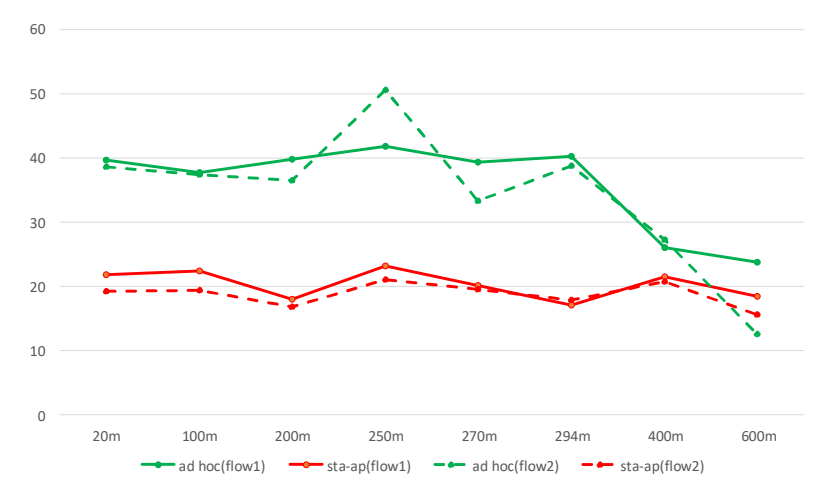

Figure 4. packets/s with different distance.

Ad hoc mode is better than STA-AP mode because STA-AP mode transfers a packet two times as long as ad hoc mode.

\section{Conclusion}

Ad hoc mode is better within a certain distance. STA-AP mode is better beyond a certain distance. After adding routing in ad hoc mode, which is better depends on routing protocol. Ad hoc mode is better than STA-AP mode because STA-AP mode transfers a packet two times as long as ad hoc mode.

In addition, ad hoc networks are very fast. Throughput rate is twice as fast as STA-AP networks. But limited wireless transmission range is the fact. By comparison, infrastructure networks can connect to a wired network and extend your wireless network's range. In the future, we will explore more complicated scenarios to get better 
comparison between ad hoc mode and infrastructure mode.

\section{References}

1. Morteza M. Zanjireh; Hadi Larijani (May 2015). A Survey on Centralised and Distributed Clustering Routing Algorithms for WSNs (PDF). IEEE 81st Vehicular Technology Conference. Glasgow, Scotland. doi:10.1109/VTCSpring.2015.7145650.

2. Morteza M. Zanjireh; Ali Shahrabi; Hadi Larijani (2013). ANCH: A New Clustering Algorithm for Wireless Sensor Networks (PDF). 27th International Conference on Advanced Information Networking and Applications Workshops. WAINA 2013. doi:10.1109/WAINA.2013.242.

3. Chai Keong Toh, Ad Hoc Mobile Wireless Networks. United States: Prentice Hall Publishers (2002).

4. Chris Hoffman, What's the Difference Between AdHoc and Infrastructure Mode Wi-Fi? https://www.howtogeek.com/180649/htg-explainswhats-the-difference-between-ad-hoc-andinfrastructure-mode/ (2016)

5. https://www.nsnam.org/docs/release/3.29/tutorial/ht ml/introduction.html\#about-ns3

6. Rakesh Kumar Jha, Pooja Kharga; Advanced Open Source Simulator: NS-3, International Journal of Computer Sciences and Engineering 3,67 (2015). 